\title{
Pseudoscalar flavor-singlets and staggered fermions
}

\author{
Eric B. Gregory*, Alan C. Irving, Christopher M. Richards \\ Theoretical Physics Division, Department of Mathematical Sciences, University of Liverpool, \\ Liverpool L69-7ZL, United Kingdom \\ E-mail: egregory@liverpool.ac.uk, aci@liv.ac.uk, cmr@liv.ac.uk
}

\section{Craig McNeile}

Department of Physics and Astronomy, The Kelvin Building, University of Glasgow, Glasgow G12-8QQ, United Kingdom

E-mail: c.mcneile@physics.gla.ac.uk

\begin{abstract}
The Asqtad improved staggered fermion formalism has been a valuable tool in successfully calculating the non-singlet parts of the hadronic spectrum. We are engaged in a project to calculate the spectrum of the pseudoscalar singlet mesons with 2+1-flavor Asqtad staggered gauge configurations. Propagators of flavor-singlet states incorporate contributions from both disconnected and connected diagrams, and hence are sensitive to any differences in the actions governing the sea and valence fermions on the lattice. As such, they also present the possibility of a probe of the validity of the "fourth-root trick" in the staggered fermion formulation. We present an update on our progress toward measuring the $\eta^{\prime}$ mass on $2+1$-flavor Asqtad staggered gauge configurations, including a review of methods and preliminary results. We also show a strong correlation between $\operatorname{Tr}\left(\gamma_{5} \otimes 1\right)$ and the topological charge in these configurations, as predicted by the index theorem.
\end{abstract}

XXIV International Symposium on Lattice Field Theory

July 23-28 2006

Tucson Arizona, US

* Speaker. 


\section{Introduction}

The pseudoscalar flavor-singlet meson system is of theoretical interest for a variety of reasons. It differs from the non-singlet by the inclusion of disconnected diagrams in its propagator, to which is attributed the mass difference between the pion and the $\eta^{\prime}$ meson $(958 \mathrm{MeV})[1,2]$. A thorough lattice QCD calculation of the spectrum of the pseudoscalar flavor-singlet system is needed for full comprehension of this connection. To date there have been a number of lattice studies of $N_{f}=2$ flavor singlet mesons[3, 5, 6, 7, 8, 9, 10,11, 12]. These proceedings contain a report on an $N_{f}=2+1$ flavor Wilson lattices fermion study from CPPACS [13].

We are engaged in a study of the pseudoscalar singlet system with $N_{f}=2+1$ flavors of improved staggered quarks. The motivation is two-fold. First, the improved staggered "Asqtad" formulation has a formidable track record of accurate reproduction and prediction of experimentally measurable quantities, in part due to the relative high speed of staggered fermion simulation and the accessibility of relatively light quark masses[4]. Second, the staggered formulation has been plagued by theoretical questions regarding the validity of the "fourth-root trick" employed in simulations where $N_{f} \neq 4$. These concerns center on whether or not the $\operatorname{det}^{1 / 4}$ of the native four-flavor staggered fermionic matrix introduces pathologies into the sea quarks. An additional motivation, then, is to see whether singlet propagators, which are uniquely sensitive to the fermionic sea, illuminate any such pathologies.

For $N_{f}$ degenerate flavors of quarks, the full propagator is

$$
G_{\eta^{\prime}}\left(x^{\prime}, x\right)=\left\langle\sum_{i} \bar{q}_{i}\left(x^{\prime}\right)\left(\gamma_{5} \otimes \mathbf{1}\right) q_{i}\left(x^{\prime}\right) \sum_{j} \bar{q}_{j}(x)\left(\gamma_{5} \otimes \mathbf{1}\right) q_{j}(x)\right\rangle,
$$

where the $\gamma_{5} \otimes 1$ denotes that the meson has $\gamma_{5}$ Dirac spinor structure, and is a singlet in staggered "taste" space. This expression includes $N_{f}$ connected terms (pion propagators):

$$
\langle\sum_{i} \overbrace{\bar{q}_{i}\left(x^{\prime}\right)\left(\gamma_{5} \otimes \mathbf{1}\right) \underbrace{q_{i}\left(x^{\prime}\right) \sum_{j} \bar{q}_{j}(x)\left(\gamma_{5} \otimes \mathbf{1}\right) q_{j}}(x)}(,
$$

and $N_{f}^{2}$ disconnected terms:

$$
\langle\sum_{i} \overbrace{\bar{q}_{i}\left(x^{\prime}\right)\left(\gamma_{5} \otimes \mathbf{1}\right) q_{i}}\left(x^{\prime}\right) \overbrace{\sum_{j}} \overbrace{\bar{q}_{j}(x)\left(\gamma_{5} \otimes \mathbf{1}\right) q_{j}}(x)\rangle .
$$

In other words

$$
G_{\eta^{\prime}}\left(x^{\prime}, x\right)=N_{f} C\left(x^{\prime}, x\right)-N_{f}^{2} D\left(x^{\prime}, x\right),
$$

where the additional fermion loop in each of the disconnected diagrams gives rise to the negative sign.

As the connected correlators are the propagators for the non-singlet meson we expect, when the ground state dominates, for them to decay exponentially in time separation on a Euclidean lattice:

$$
G_{\pi}(t)=C(t) \sim e^{-m_{\pi} t}
$$

Furthermore,

$$
G_{\eta^{\prime}}(t)=N_{f} C(t)-N_{f}^{2} D(t) \sim e^{-m_{\eta^{\prime}} t}
$$


Then the ratio of disconnected to connected contributions should be

$$
R(t)=\frac{N_{f}^{2} D(t)}{N_{f} C(t)}=1-A e^{-\left(m_{\eta^{\prime}}-m_{\pi}\right) t},
$$

if the sea quarks are appropriately dynamical. In the case of quenched QCD we expect instead $[14,15]$

$$
R(t) \sim A+B t
$$

It is also possible that the $\operatorname{det}^{1 / 4}$ in the staggered formulation introduces other pathologies that may manifest themselves in this ratio.

Measuring disconnected correlators is a difficult task. They are noisier than connected correlators, as they contain fluctuations of the fermionic sea. Hence one must use some sort of all-to-all propagators to get as much information out of a particular gauge configuration as possible. Additionally, the pseudoscalar singlet is closely related to very slow topological modes, necessitating the use of very long time series. We will describe how we address these challenges below.

\section{Simulation and Measurement}

We have at our disposal the large publicly-available library of MILC Asqtad lattices. Our preliminary study has predominately utilized the "coarse lattice" ensembles, with lattices of $20^{3} \times 64$ and lattice spacing of $a \approx 0.125 \mathrm{fm}[16]$. The typical ensemble has between 400 to 600 configurations separated by 6 trajectories.

On each gauge configuration we measure the connected correlators with standard point sources with routines integrated into the Chroma code [17]. We measure disconnected correlators with stochastic volume-filling sources[18, 19, 20], $\eta(x)$. Given that on averaging over sources

$$
\delta_{x, x^{\prime}}=\left\langle\eta(x)^{\dagger} \eta(x)\right\rangle_{\eta},
$$

and defining $M \phi=\eta$, then the pseudoscalar loop operator is

$$
\mathscr{O}_{\gamma_{5} \otimes \mathbf{1}}(t)=\sum_{x_{4}=t}\left\langle\phi^{\dagger}(x) \Delta_{\gamma_{5} \otimes \mathbf{1}} \eta(x)\right\rangle_{\eta} .
$$

The operator $\Delta_{\gamma_{5} \otimes \mathbf{1}}$ is the appropriate four-link covariant shift, displacing the quark and antiquark to opposite corners of the hypercube, with appropriate Kogut-Susskind phasing to effect the $\gamma_{5} \otimes \mathbf{1}$ operator. We average over $N_{s}=64$ noise sources. The disconnected correlator is then:

$$
D(t)=\frac{1}{T} \sum_{t^{\prime}} \mathscr{O}\left(t^{\prime}\right) \mathscr{O}\left(t^{\prime}+t\right)
$$

In practice one can do better than Equation 2.2. Venkataraman and Kilcup[10] describe a variance reduction (VKVR) trick applicable to the four-link pseudoscalar singlet operator. Recognizing that the Asqtad $\not D$ connects only odd sites to even sites, and the $M^{\dagger} M=\left(D^{2}+m^{2}\right)$ and its inverse connects even to even and odd to odd only then:

$$
m\left\langle\phi^{\dagger} \Delta_{\gamma_{5} \otimes 1} \phi\right\rangle=\left\langle\phi^{\dagger} \Delta_{\gamma_{5} \otimes 1} \eta\right\rangle,
$$


with the left-hand expression having a minimized variance. The equivalence of the expectation value depends on the $\Delta_{\gamma_{5} \otimes 1}$ operator separating the source and sink by an even number of links (four).

The numerical work by the TrinLat group suggests that using a set of dilute noise sources, each defined on a subset of the lattice but collectively spanning the lattice, can reduce the variance of measured propagators[20]. In the case of disconnected correlators for pseudoscalar singlets with Asqtad fermions, we found no discernible advantage.

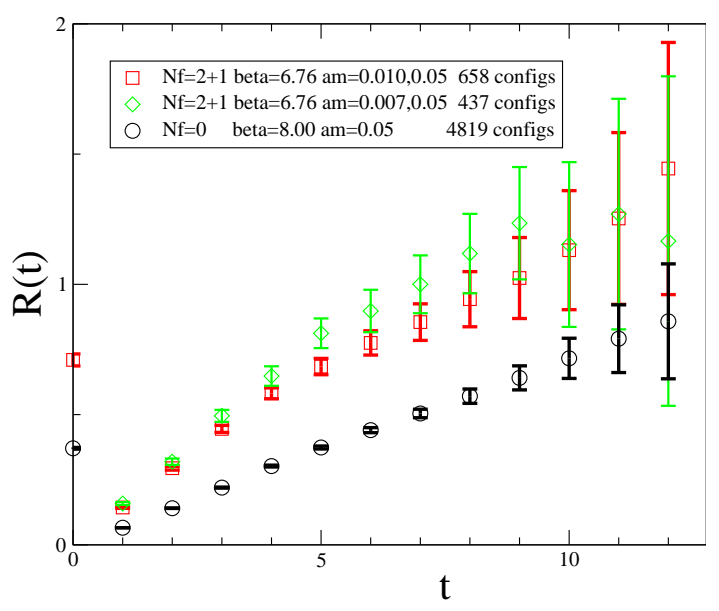

Figure 1: A comparison of $D / C$ ratios for quenched and $N_{f}=2+1$ flavor configurations. The $\beta=2.76$ $a m=0.007$ points were computed with only 40 noise sources per configuration and without the VKVR trick.

\section{Analysis}

We were able to extract signals for connected and disconnected correlators for several ensembles of MILC coarse lattices. For $N_{f}=2+1$ configurations with two degenerate flavors of light sea quarks and one strange sea quark flavor, expression 1.7 generalizes to[21]

$$
R(t)=\frac{4 D_{q q}(t)+4 D_{q s}(t)+D_{s s}(t)}{2 C_{q q}(t)+C_{s s}(t)} .
$$

The disconnected correlators, $D_{q q}, D_{q s}$ and $D_{s s}$, are formed with two light quark loop operators, with a light loop operator and a strange loop operator, and with two strange loop operators, respectively. The connected correlators $C_{q q}$ and $C_{s s}$ are light quark and strange quark correlators, respectively.

When we form the $D / C$ ratio for $N_{f}=2+1$ lattices we get a curve marginally consistent with a plateau at one as in Equation 1.7. However, it is also consistent with a linear relationship such as that in Equation 1.8. With the MILC quenched ensemble ( $\beta=8.0 \mathrm{am}=0.050,408$ configs), we find a $D / C$ curve that is inconsistent with a linear relationship as in Equation 1.8. See Figure 1. 


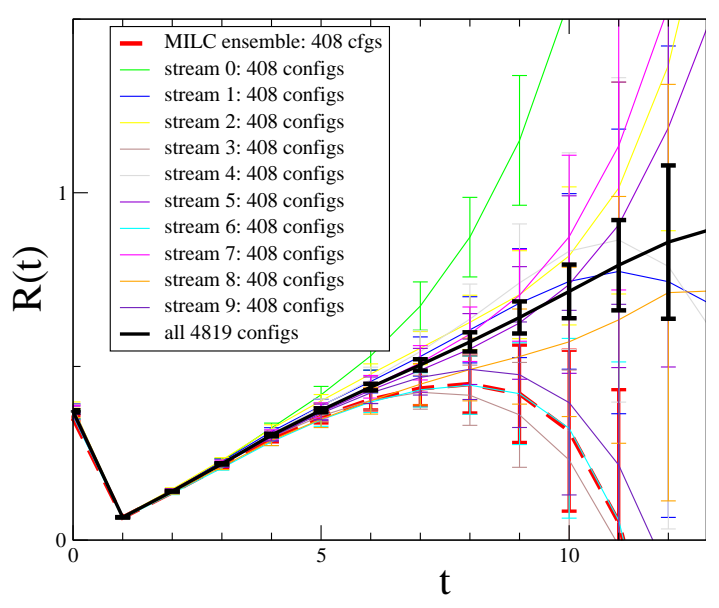

Figure 2: $\quad D / C$ ratio for $4819 \beta=8.00, a m=0.05$, quenched configurations, and for subsets of 408 configurations, including the original MILC ensemble.

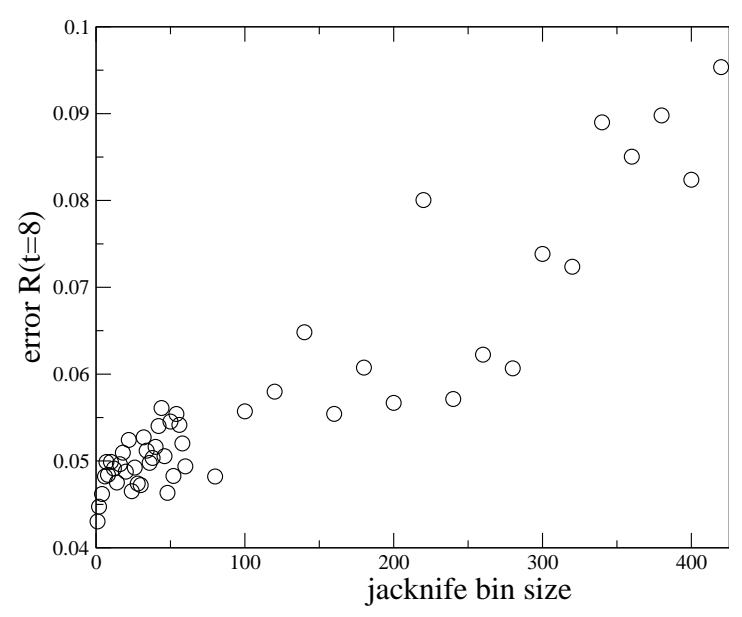

Figure 3: Error on $R(t=8)$ for $4819 \beta=8.00$, $a m=0.05$, quenched configurations, as a function of jackknife bin size.

We hypothesized that the $\gamma_{5} \otimes \mathbf{1}$ loop operator might be subject to the particularly long autocorrelation times of slow topological charge modes. As quenched configurations are relatively inexpensive to produce, we have extended the MILC ensemble by nearly a factor of 12 to 4819 configurations. When we analyze the entire ensemble, the $R(t)$ curve is more consistent with Equation 1.8 (see Figure 2). However, subsets of 408 configurations are often mutually inconsistent by a margin of two standard deviations even when determined with relatively large jackknife bin size — far greater than the integrated autocorrelation time of about 10 configurations. We interpret this 
to mean that the time series autocorrelation does not decay as a single exponential, and that there are autocorrelation time scales in this mode that are as long as 100 configurations or more.

\section{Topological Charge}

Using the Atiyah-Singer index theorem [22], Smit and Vink [23] equate a fermionic expression for the topological charge

$$
Q=m \kappa_{p}\left\langle\operatorname{Tr}\left(\gamma_{5} M^{-1}\right)\right\rangle_{U}
$$

with the traditional gluonic definition:

$$
Q(x)=\frac{g^{2}}{64 \pi^{2}} \varepsilon^{\mu v \rho \sigma} F_{\mu v}^{a}(x) F_{\rho \sigma}^{a}(x)
$$

Equation 4.1 is merely the loop operator in Equation 2.2, integrated over time. In [24], Alles et al. show the equivalence of these two definitions on Wilson configurations. As a cross check of our loop operator, we measured the topological charge using the gluonic definition, Equation 4.2, using hypercubic blocking[25] implemented in the MILC code [26]. We found a strong linear correlation between the integrated loop operator and the topological charge as defined gluonically in Equation 4.2. (See Figure 4 for an example.)

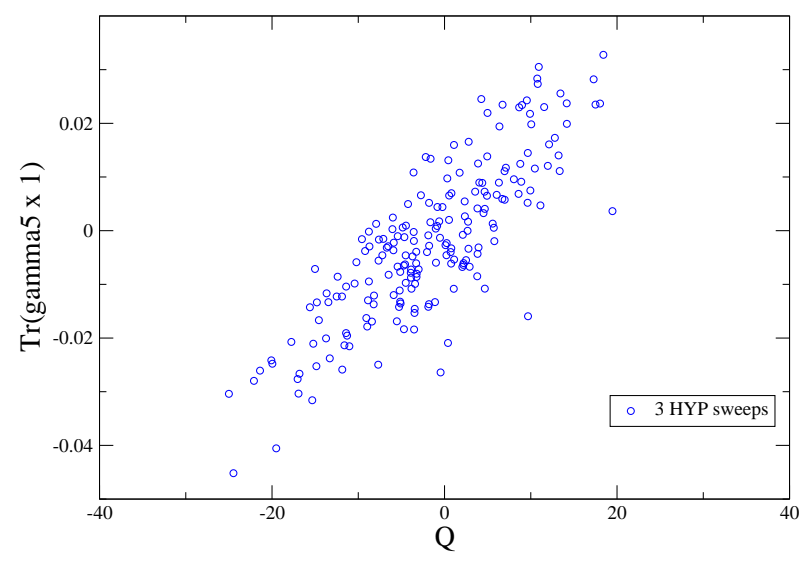

Figure 4: Fermionic versus gluonic topological charge (after three HYP cooling sweeps) for $N_{f}=2+1$ $\beta=6.76 \mathrm{am}=0.01,0.05$. Normalizations not consistent between the axes.

\section{Conclusions}

We interpret these results to mean that there is potential in Asqtad fermions as an avenue for investigating pseudoscalar singlet systems. If our experience with quenched configurations is a guide, one can achieve good signals for disconnected propagators with the order of $10^{4}$ trajectories. While this is far in excess of any current MILC Asqtad ensembles, we are currently in the process 
of generating such a $\sim 2 \times 10^{4}$ trajectory ensemble on two racks of a QCDOC machine. Use of the VKVR trick has proven to be invaluable for reducing the variance of disconnected $\gamma_{5} \otimes \mathbf{1}$ operators. Careful measurement of a suite of fuzzed operators may also help in extracting the pseudoscalar singlet system spectrum for $N_{f}=2+1$ staggered QCD, as well as shed light on the validity of the fourth-root trick.

\section{References}

[1] E. Witten, Nucl. Phys. B 156 (269).

[2] G. Veneziano, Nucl. Phys. B 159 (213).

[3] S. Itoh, Y. Iwasaki and T. Yoshie, Phys. Rev. D 36, (527).

[4] C. Aubin et al., Phys. Rev. D 70 (094505) [ arXiv:hep-lat/0402030].

[5] C. McNeile and C. Michael [UKQCD Collaboration], Phys. Lett. B 491 (123) [Erratum-ibid. B 551, 391 (2003)] [ arXiv:hep-lat/0006020].

[6] T. Struckmann et al. [TXL Collaboration], Phys. Rev. D 63 (074503) [ arXiv:hep-lat/0010005].

[7] V. I. Lesk et al. [CP-PACS Collaboration], Phys. Rev. D 67 (074503) [arXiv:hep-lat/0211040].

[8] K. Schilling, H. Neff and T. Lippert, [ arXiv:hep-lat/0401005].

[9] T. DeGrand and U. M. Heller [MILC collaboration], Phys. Rev. D 65 (114501) [ arXiv:hep-lat/0202001].

[10] L. Venkataraman and G. Kilcup, [ arXiv:hep-lat/9711006].

[11] J. B. Kogut, J. F. Lagae and D. K. Sinclair, Phys. Rev. D 58 (054504) [arXiv:hep-lat/9801020].

[12] H. Fukaya and T. Onogi, Phys. Rev. D 70, (054508)[arXiv:hep-lat/0403024].

[13] CP-PACS et al. [JLQCD Collaborations], [arXiv:hep-lat/0610021].

[14] C. W. Bernard and M. F. L. Golterman, Phys. Rev. D 46, (853) [arXiv:hep-lat/9204007].

[15] C. W. Bernard and M. F. L. Golterman, Phys. Rev. D 49, (486) [arXiv:hep-lat/9306005].

[16] C. W. Bernard et al., Phys. Rev. D 64, (054506). [arXiv:hep-lat/0104002].

[17] R. G. Edwards and B. Joo [SciDAC Collaboration], Nucl. Phys. Proc. Suppl. 140, 832 (2005) [arXiv:hep-lat/0409003].

[18] J. F. Markham and T. D. Kieu, Nucl. Phys. Proc. Suppl. 94 (813) [arXiv:hep-lat/0011001].

[19] F. Farchioni, G. Muenster and R. Peetz, Eur. Phys. J. C, 38 (329) [arXiv:hep-lat/0404004].

[20] J. Foley, K. Jimmy Juge, A. O'Cais, M. Peardon, S. M. Ryan and J. I. Skullerud, [arXiv:hep-lat/0505023].

[21] E. B. Gregory, A. C. Irving, C. McNeile, S. Miller and Z. Sroczynski, PoS LAT2005, (083) [arXiv:hep-lat/0509193].

[22] M. F. Atiyah and I. M. Singer, Annals Math. 87, (484).

[23] J. Smit and J. C. Vink, Nucl. Phys. B 286, (485).

[24] B. Alles et al., Phys. Rev. D 58, (071503) [arXiv:hep-lat/9803008].

[25] C. Aubin et al. [MILC Collaboration], Nucl. Phys. Proc. Suppl. 140, (600) [arXiv:hep-lat/0409051].

[26] http://physics.utah.edu/ detar/milc.html 